University of Nebraska - Lincoln

DigitalCommons@University of Nebraska - Lincoln

8-2006

\title{
Magnetic Moment Softening and Domain Wall Resistance in $\mathrm{Ni}$ Nanowires
}

John D. Burton

University of Nebraska-Lincoln, jburton2@unl.edu

Renat F. Sabirianov

University of Nebraska at Omaha, rsabirianov@mail.unomaha.edu

Sitaram S. Jaswal

University of Nebraska-Lincoln, sjaswal1@unl.edu

Evgeny Y. Tsymbal

University of Nebraska-Lincoln, tsymbal@unl.edu

O. N. Mryasov

Seagate Research, Pittsburgh, PA

Follow this and additional works at: https://digitalcommons.unl.edu/physicstsymbal

Part of the Condensed Matter Physics Commons

Burton, John D.; Sabirianov, Renat F.; Jaswal, Sitaram S.; Tsymbal, Evgeny Y.; and Mryasov, O. N., "Magnetic Moment Softening and Domain Wall Resistance in Ni Nanowires" (2006). Evgeny Tsymbal Publications. 4.

https://digitalcommons.unl.edu/physicstsymbal/4

This Article is brought to you for free and open access by the Research Papers in Physics and Astronomy at DigitalCommons@University of Nebraska - Lincoln. It has been accepted for inclusion in Evgeny Tsymbal Publications by an authorized administrator of DigitalCommons@University of Nebraska - Lincoln. 


\title{
Magnetic Moment Softening and Domain Wall Resistance in Ni Nanowires
}

\author{
J. D. Burton, ${ }^{1,3}$ R. F. Sabirianov, ${ }^{2,3}$ S. S. Jaswal, ${ }^{1,3}$ and E. Y. Tsymbal ${ }^{1,3, *}$ \\ ${ }^{1}$ Department of Physics and Astronomy, University of Nebraska, Lincoln, Nebraska 68588-0111, USA \\ ${ }^{2}$ Department of Physics, University of Nebraska, Omaha, Nebraska 68182-0266, USA \\ ${ }^{3}$ Nebraska Center for Materials and Nanoscience, University of Nebraska, Lincoln, Nebraska 68588-0111, USA \\ O. N. Mryasov \\ Seagate Research, Pittsburgh, Pennsylvania 15222, USA \\ (Received 27 January 2006; published 18 August 2006)
}

\begin{abstract}
We perform $a b$ initio calculations of the electronic structure and conductance of atomic-size Ni nanowires with domain walls only a few atomic lattice constants wide. We show that the hybridization between noncollinear spin states leads to a reduction of the magnetic moments in the domain wall resulting in the enhancement of the domain wall resistance. Experimental studies of the magnetic moment softening may be feasible with modern techniques such as scanning tunneling spectroscopy.
\end{abstract}

DOI: 10.1103/PhysRevLett.97.077204

PACS numbers: 75.47.Jn, 72.25.Ba, 73.63.-b, 75.75.+a

The study of domain walls (DWs) in bulk ferromagnetic materials began in the early 20th century with contributions from Bloch [1], Landau and Lifshitz [2], and Néel [3]. In bulk $3 d$ metal ferromagnets DWs are wide $(\sim 100 \mathrm{~nm})$ on the scale of the lattice spacing due to the strong exchange interaction which tends to align neighboring regions of magnetization, compared to anisotropic effects which prefer the magnetization to lie along specific directions. With the recent interest in magnetic nanostructures it has been shown that DWs can be quite thin due to the enhanced effective anisotropy of constricted geometry in nanowires and nanocontacts [4]. Extremely narrow DWs with a width of the order of a lattice constant have recently been observed in Fe nanowires grown on W [5] and Mo [6].

The DW width controls the DW resistance. The origin of the DW resistance is known to be the mixing of up- and down-spin electrons due to the mistracking of the electron's spin in passing through the DW [7]. The narrower DW width results in a larger angle between the magnetization directions of successive atomic layers thereby lowering the electron transmission. In bulk ferromagnets DWs do not affect appreciably the resistance because the DW width is much larger than the Fermi wave length, and hence electrons can follow adiabatically the slowly varying magnetization direction within the DW. In magnetic nanoconstrictions, where a DW can be very narrow, the DW resistance may be appreciable.

Experimental studies of such DWs are feasible due to remarkable progress in experimental techniques, making possible to synthesize and characterize structures at the atomic scale. In particular, conducting nanowires can be fabricated by breaking metal junctions [8], by pulling apart two metal surfaces [9], or by electrodepositing on prepatterned electrodes [10]. Using these techniques an atomic scale DW can be formed in magnetic nanowires and studied by modern experimental techniques such as scan- ning tunneling spectroscopy [11]. Electronic transport across DWs exhibits interesting spin-dependent phenomena recently reviewed by Marrows [12].

The theoretical description of the DW resistance so far was based on either free-electron models [13] in which the DW is represented by an appropriate potential profile or first-principles calculations $[14,15]$ in which the DW is typically described by a spin-spiral structure. All these models assume that the DW is rigid; i.e., they neglect any spatial variation of the magnitude of the magnetic moment across the DW. It is well established, however, that the magnitude of the magnetic moments in itinerant magnets can depend strongly on the orientation of the neighboring moments [16]. This effect is relatively weak in well localized ferromagnets like $\mathrm{Fe}$, but can reduce and even destroy the atomic magnetic moment of the itinerant ferromagnets, like Ni.

The origin of this phenomenon is the hybridization between noncollinear spin states. In the uniformly magnetized material with no spin-orbit coupling the minorityand majority-spin bands are independent. However, in a noncollinear state, such as a DW, this is no longer the case and the two spin bands are hybridized. This spin mixing leads to charge transfer and level broadening, which results in the reduction of the overall exchange splitting between majority- and minority-spin states on each atom, and hence the atomic moments are reduced. In bulk DWs of any ferromagnetic material, this effect is small due to the slow variation of the direction of the magnetization. For the atomic scale DWs, however, there is a large degree of canting between neighboring magnetic moments. This leads to a significant hybridization between spin states which leads to a reduction, or softening, of the magnetic moments within the constrained DW. The magnetic moment softening affects the DW resistance due to the local perturbation in the electronic potential. 
We note that the spatial variation of the magnetization in DWs has been suggested before, but only in the context of finite temperature magnetic disorder of the (fixed magnitude) atomic magnetic moments in a DW [17]. The effect of spatial variation of the magnetization on DW resistance was addressed previously, but only within the diffusive transport regime and a free-electron model [18].

In this Letter we illustrate the importance of magnetic moment softening by performing $a b$ initio calculations of the electronic structure and ballistic conductance of atomic scale DWs in Ni nanowires. We show that the magnetic moments within DWs only a few lattice constants wide can be significantly reduced compared to the magnetic moments in a uniformly magnetized wire due to the presence of substantial hybridization between spin states. We find that the magnetic moment softening strongly enhances the DW resistance due to additional scattering resulting from the local perturbation in the electronic potential.

Density functional calculations of the spin-dependent electronic structure of atomic scale DWs in Ni nanowires were performed using the tight-binding linear muffin-tinorbital method [19] in the atomic sphere approximation and the local spin density approximation for the exchangecorrelation energy. We used the real space recursion method [20] with a Beer-Pettifor terminator [21] to calculate the local density of states (DOS). Ultrathin domain walls were examined for two different freestanding $\mathrm{Ni}$ wires based on the bulk fcc structure: (110) monatomic chains and $5 \times 4$ wires (described below). All the structures used the lattice constant $a=3.52 \AA$ of bulk fcc Ni.

In the calculations we constructed a central region containing the DW, surrounded by two uniformly magnetized leads aligned antiparallel relative to each other. The selfconsistent calculations were carried out for the central region and a large enough portion of the surrounding leads so that the outermost atoms of the self-consistent region were similar to those in the remaining semi-infinite section of the leads in the uniformly magnetized state. A DW was modeled by a finite spin spiral with the relative angle between neighboring atomic layers of magnetic moments being $180^{\circ} /(N+1)$, where $N$ is the number of atomic layers in the DW [22].

First, we consider an $N=1 \mathrm{DW}$ in a monatomic Ni wire [Fig. 1(a)]. Figure 1 displays the results of our calculations for the $N=1 \mathrm{DW}$. The electronic potentials of the antiparallel magnetized lead sites were frozen, while on the central five sites the electronic structure was calculated self-consistently. We find a $16 \%$ reduction of the magnetic moment on the central site and a $7 \%$ reduction on the two neighboring sites. The reduction in the magnetic moment on the central site is due to the hybridization with the different spin states of the noncollinear neighbors, which results in the reduction of the exchange splitting, as described earlier. This fact is evident from Fig. 1(b), which shows the local DOS for the uniformly magnetized monatomic Ni wire and for the central atom of an $N=1 \mathrm{DW}$.
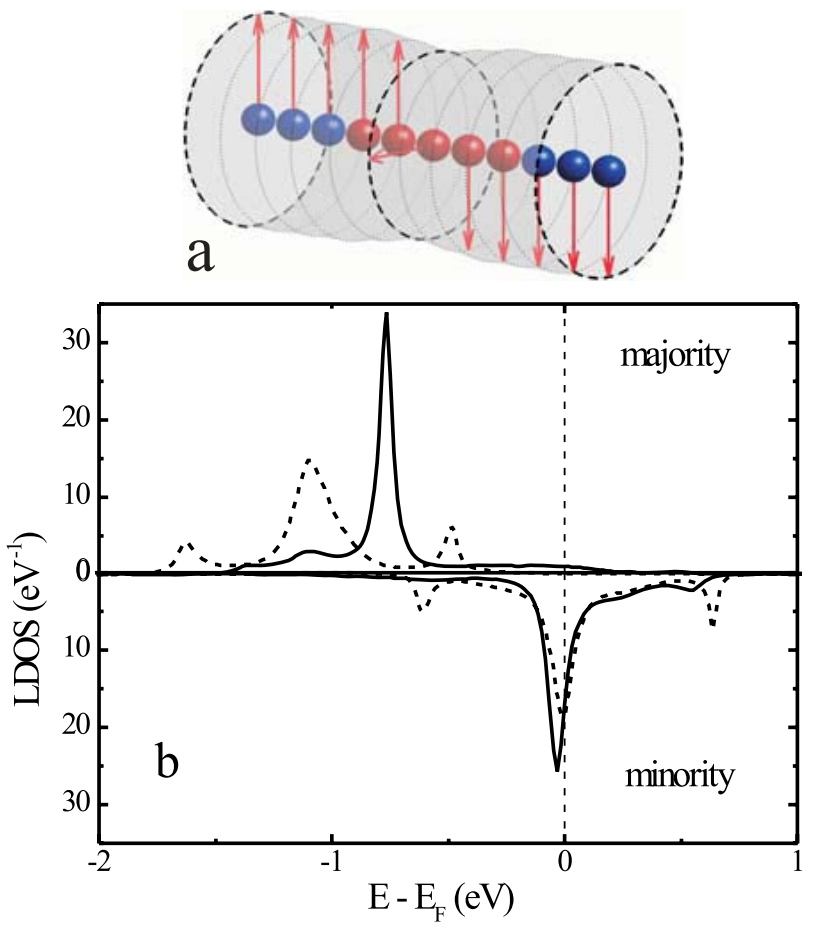

FIG. 1 (color online). (a) A monatomic wire with an $N=1$ DW. The arrows show the orientation and relative magnitude of calculated magnetic moments. (b) DOS per atom of the uniformly magnetized monatomic wire (dashed curve) and the local DOS (LDOS) at the center of an $N=1 \mathrm{DW}$ (solid curve).

The reduction in the moment of the nearest neighbors of the central atom is only about half of that of the central atom because they are noncollinear with only one of the two neighbors. Also apparent in Fig. 1(b) is an overall broadening of the DOS on the central site compared to the uniformly magnetized state produced by the spin mixing.

Figure 2 shows the magnetic moment profile for several DW widths in the monatomic wire. The reduction in the moment is strongly dependent on the width of the DW. For $N=0$ and $N=1$ DWs, the effect of softening is the largest owing to the fact that the degree of noncollinearity is the largest in these two cases. As the width of the DW increases the softening decreases because of the reduction of the angle between the nearest neighbor magnetic moments.

Figure 3(a) shows the $5 \times 4$ wire which has five atoms in one layer and four in the next layer resulting in three nonequivalent sites, each indicated by a different color. Although the average magnetic moment of the uniformly magnetized $5 \times 4$ wire, $\mu=0.7 \mu_{B}$, is lower than that of the monatomic wire, $\mu=1.1 \mu_{B}$, we find that in the presence of ultrathin DWs the spatial variation of the magnetization displays qualitatively similar behavior. Figure 3(b) shows the magnetic moments found for three DW widths in the $5 \times 4$ wire. The largest reduction in magnetic moment is nearly $90 \%$ for the central site of the $N=1 \mathrm{DW}$, and the effect is still substantial for the $N=5 \mathrm{DW}$ where the 


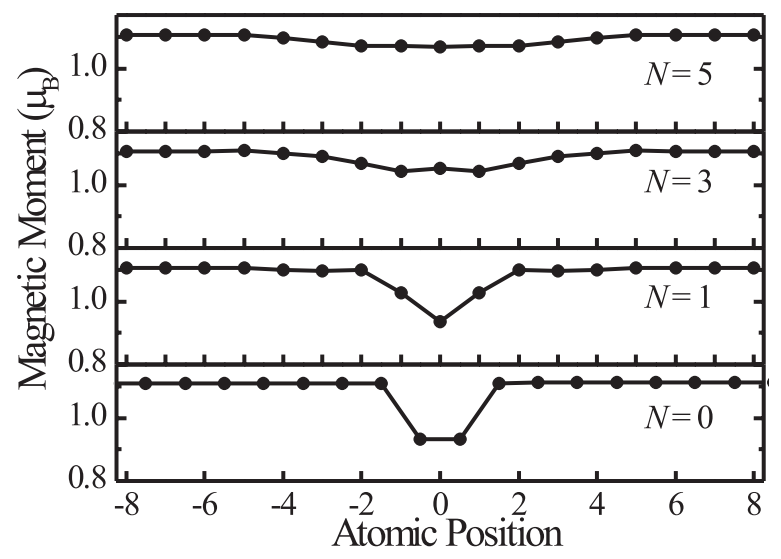

FIG. 2. Magnetic moments in the monatomic Ni wire as a function of distance from the DW center for several DW widths. The $N=1$ plot corresponds to Fig. 1(a).

magnetic moment of the central layer is softened by about $10 \%$. The effect is significantly larger in the $5 \times 4$ wire than that in the monatomic chain due to the enhanced hybridization reflecting an increase in the number of neighboring atoms.

The magnetic moment softening affects dramatically the conductance across DWs. We calculate the conductance of the $5 \times 4 \mathrm{Ni}$ wire with an $N=1 \mathrm{DW}$ using the standard technique described in detail in Ref. [15]. It should be

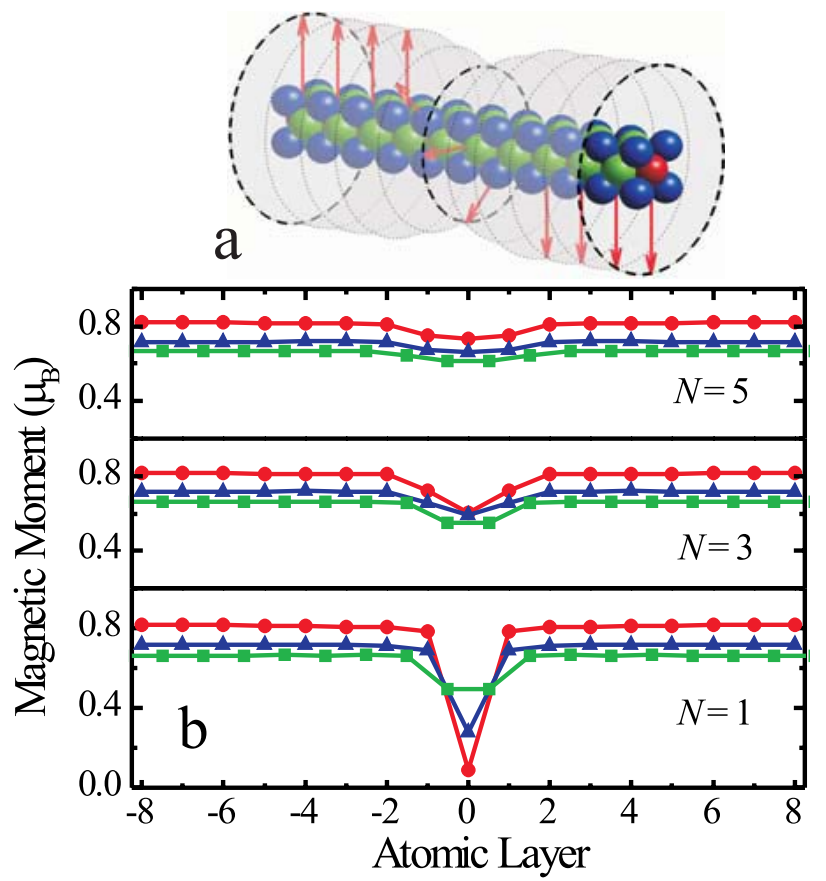

FIG. 3 (color online). (a) The $5 \times 4$ nanowire showing the three nonequivalent sites and an $N=3$ DW. Each arrow represents the magnitude and orientation of the average magnetic moment of the plane with 5 atoms. (b) The self-consistent magnetic moments for several DW widths. The color in the plot corresponds to the site of the same color in (a). noted, however, that in Ref. [15] the Hamiltonian for the DW region was built by simply rotating (in spin space) the self-consistent potentials obtained for the uniformly magnetized wire. Here we derive the Hamiltonian from the self-consistent potentials found in the presence of the abrupt DW. For comparison we also calculate the conductance in the same way as Ref. [15], which allows us to determine the contribution of magnetic moment softening to the DW resistance.

Figure 4(a) shows the spin-resolved conductance, $G_{\mathrm{FM}}$, of a uniformly magnetized $5 \times 4 \mathrm{Ni}$ wire as a function of energy $E$. The conductance is quantized in steps of $e^{2} / h$, corresponding to the number of bands crossing the energy $E$. The bands are seen in Fig. 4(b) as being bound by the sharp peaks corresponding to the band edges. The conductance in the zero-bias limit is given by the value at the Fermi energy, $E_{\mathrm{F}}$, located at the center of the narrow minority band. For the $5 \times 4 \mathrm{Ni}$ wire it is $14 e^{2} / h$. Figure 4(c) displays the conductance through an $N=1$ $\mathrm{DW}, G_{\mathrm{DW}}$. For a "rigid" DW (no softening of the magnetic moments) the Hamiltonian is constructed from the selfconsistent potentials of the uniformly magnetized wire, while for the "soft" DW we use the potentials that yield reduced magnetic moments in the DW region, as seen in Fig. 3. We find that the magnetic moment softening in the DW leads to a reduction in the conductance at the Fermi energy from $5.38 e^{2} / h$ to $3.21 e^{2} / h$.

The origin of this phenomenon can be understood using a simple tight-binding model of a monatomic chain with an abrupt $N=0 \mathrm{DW}$. We model the majority states by a wide band, characterized by the (large) nearest neighbor hop-

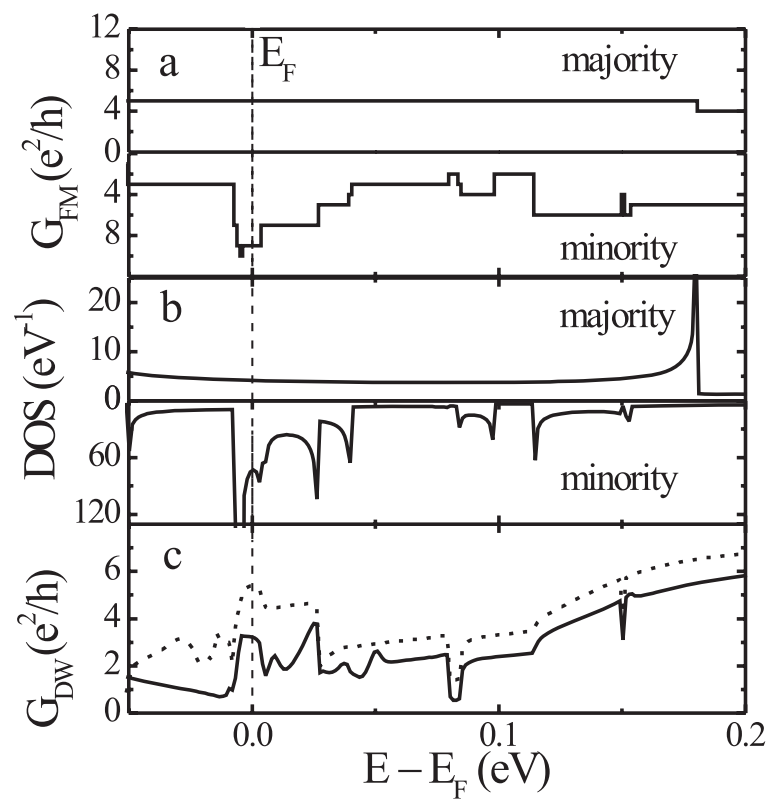

FIG. 4. (a) Spin-resolved conductance $G_{\mathrm{FM}}$ and (b) density of states near the Fermi energy $E_{\mathrm{F}}$ as a function of energy for the uniformly magnetized $5 \times 4 \mathrm{Ni}$ wire. (c) Conductance of the $N=1 \mathrm{DW}, G_{\mathrm{DW}}$, as a function of energy for the rigid DW (dashed curve) and for the soft DW (solid curve). 


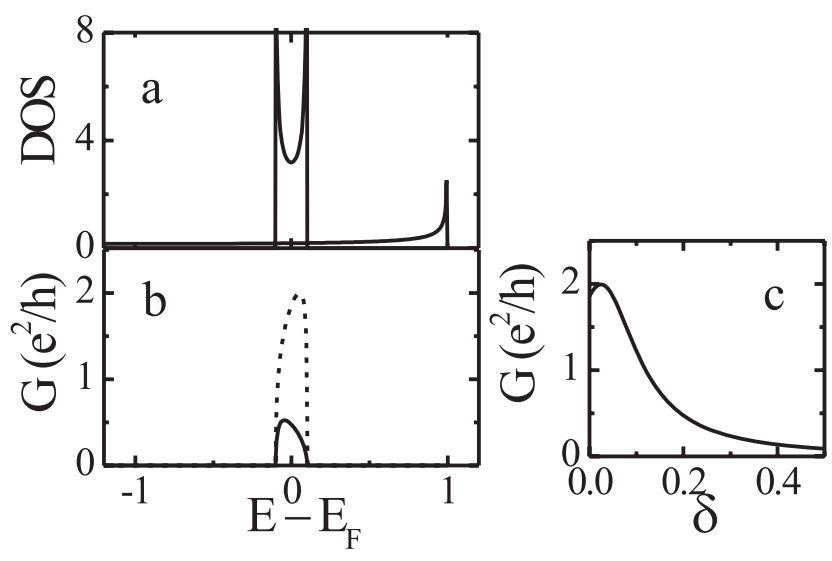

FIG. 5. Results of a simple tight-binding model. (a) DOS of the uniformly magnetized state versus energy for majority- (wide band) and minority- (narrow band) spin electrons. (b) Conductance versus energy for a rigid DW ( $\delta=0$, dashed line) and for a soft DW ( $\delta=0.2$, solid line). (c) Conductance vs $\delta$ for $E=E_{F}$.

ping parameter $t_{\text {maj }}$, to emulate the wide band near the Fermi energy [see Fig. 4(b)]. The minority states are modeled by a narrow band with (small) hopping $t_{\min }$, offset from the center of the majority band by energy $\Delta$. To model magnetic moment softening we assume that the on-site energies of the two interface sites are shifted relative to the uniformly magnetized leads. Majority states are shifted up by energy $\delta$, and minority states are shifted down by the same amount $\delta$. This corresponds effectively to a reduction of the local exchange splitting by $2 \delta$ on the two interface sites, and hence softening of the magnetic moment. The parameters for our model are chosen as follows: $t_{\text {maj }}=1, t_{\min }=0.05$, and $\Delta=1$. The results of the model are shown in Fig. 5. As is seen from Figs. 5(b) and $5(\mathrm{c})$, the model predicts a drastic reduction in the conductance as a result of magnetic moment softening. The origin of this reduction is the local perturbation in the electronic potential which leads to stronger scattering of transport electrons by the DW. When the on-site energies of the interface atoms are shifted with respect to the narrow minority band, which determines the energy window for conductance, these sites act as additional scatterers that hinder conductance.

Scanning tunneling spectroscopy (STS) has been shown to reveal differences in the electronic structure of domains and DWs in Fe nanowires grown on W(110) surfaces [11]. It may be possible to use techniques such as STS to study the changes in the electronic structure due to magnetic moment softening in narrow DWs predicted here. We hope, therefore, that our theoretical predictions will stimulate experimental studies of the electronic and transport properties of atomic scale domain walls.

This work is supported by Seagate Research, the NSF (Grants No. DMR-0203359 and No. MRSEC DMR0213808), and the Nebraska Research Initiative. The calculations were performed using the Research Computing
Facility of the University of Nebraska-Lincoln.

*Electronic address: tsymbal@unl.edu

[1] F. Bloch, Z. Phys. 74, 295 (1932).

[2] L. Landau and E. Lifshitz, Phys. Z. Sowjetunion 8, 153 (1935).

[3] L. Néel, J. Phys. Radium 17, 250 (1956).

[4] P. Bruno, Phys. Rev. Lett. 83, 2425 (1999).

[5] M. Pratzer et al., Phys. Rev. Lett. 87, 127201 (2001).

[6] J. Prokop, A. Kukunin, and H. J. Elmers, Phys. Rev. Lett. 95, 187202 (2005).

[7] G. G. Cabrera and L. M. Falicov, Phys. Status Solidi (b) 61, 539 (1974).

[8] N. Agraït, A. Levy Yeyati, and J. M. van Ruitenbeek, Phys. Rep. 377, 81 (2003).

[9] V. Rodrigues, J. Bettini, P. C. Silva, and D. Ugarte, Phys. Rev. Lett. 91, 096801 (2003).

[10] C.S. Yang, C. Zhang, J. Redepenning, and B. Doudin, Appl. Phys. Lett. 84, 2865 (2004).

[11] M. Bode et al., Phys. Rev. Lett. 89, 237205 (2002).

[12] C. H. Marrows, Adv. Phys. 54, 585 (2005).

[13] H. Imamura, N. Kobayashi, S. Takahashi, and S. Maekawa, Phys. Rev. Lett. 84, 1003 (2000); L. R. Tagirov, B. P. Vodopyanov, and K. B. Efetov, Phys. Rev. B 65, 214419 (2002); V. K. Dugaev, J. Berakdar, and J. Barnas, Phys. Rev. B 68, 104434 (2003); J. D. Burton et al., Appl. Phys. Lett. 85, 251 (2004).

[14] J. B. A. N. van Hoof et al., Phys. Rev. B 59, 138 (1999); J. Kudrnovsky et al., Phys. Rev. B 62, 15084 (2000); B. Yu. Yavorsky et al., Phys. Rev. B 66, 174422 (2002); J. Velev and W. H. Butler, Phys. Rev. B 69, 094425 (2004).

[15] R. F. Sabirianov et al., Phys. Rev. B 72, 054443 (2005).

[16] J. Hubbard, Phys. Rev. B 23, 5974 (1981); S. A. Turzhevskii, A. I. Likhtenshtein, and M. I. Katsnelson, Sov. Phys. Solid State 32, 1138 (1990); O. N. Mryasov, V. A. Gubanov, and A. I. Liechtenstein, Phys. Rev. B 45, 12330 (1992)

[17] L. N. Bulaevskĭ and V. L. Ginzburg, Sov. Phys. JETP 18, 530 (1964); V. A. Zhirnov, Zh. Eksp. Teor. Fiz. 35, 1175 (1959) [Sov. Phys. JETP 8, 822 (1959)]; N. Kazantseva, R. Wieser, and U. Nowak, Phys. Rev. Lett. 94, 037206 (2005).

[18] R. P. van Gorkom, A. Brataas, and G. E. W. Bauer, Phys. Rev. Lett. 83, 4401 (1999).

[19] O. K. Andersen and O. Jepsen, Phys. Rev. Lett. 53, 2571 (1984).

[20] R. Haydock, in Solid State Physics (Academic Press, New York, 1980), Vol. 35, pp. 215-294.

[21] N. Beer and D. G. Pettifor, in The Electronic Structure of Complex Systems, edited by P. Phariseau and W. M. Temmerman, NATO ASI, Ser. B, Vol. 113 (Plenum, New York, 1984).

[22] To justify this model for the DW structure we performed self-consistent noncollinear calculations of the magnetic moment directions for the $N=7 \mathrm{DW}$ in a $5 \times 4 \mathrm{Ni}$ wire. We found that the converged magnetic structure represents a DW which has magnetic moment orientations almost identical to those assumed within our model with deviations not larger than $2^{\circ}$. 